\title{
Aprendizaje servicio e innovación educativa en la Universidad de Santiago de Compostela: estrategia para su institucionalización
}

\author{
Javier Agrafojo Fernández, Beatriz García Antelo, Elisa Jato Seijas
}

Universidad de Santiago de Compostela, España

\section{Resumen}

El aprendizaje servicio constituye una propuesta de innovación educativa que optimiza los procesos de enseñanza-aprendizaje, favorece la adquisición de competencias profesionales y el fortalecimiento de un compromiso cívico-social con la comunidad. La Universidad de Santiago de Compostela (USC) ha iniciado el proceso de institucionalización del ApS, aportando consolidación y difusión a diferentes experiencias que progresivamente han ido emergiendo. En el presente trabajo se describe este desarrollo, destacando la formación del profesorado, los proyectos de innovación educativa y la difusión y reconocimiento como los tres pilares que sustentan la estrategia de institucionalización del ApS en la USC. Los resultados obtenidos hasta el momento ponen de manifiesto el interés de un número importante de profesorado sobre esta metodología y su aportación para la formación del alumnado.

\section{Palabras clave}

Aprendizaje servicio, innovación educativa, docencia, universidad, institucionalización.

Fecha de recepción: 9/II/2017

Fecha de aceptación: 9/III/2017

Agrafojo, J.; García, B.; Jato, E. (2017). Aprendizaje servicio e innovación educativa en la Universidad de Santiago de Compostela: estrategia para su institucionalización. RIDAS, Revista Iberoamericana de Aprendizaje Servicio, 3, 23-34. DOI10.1344/RIDAS2017.3.3 


\title{
Service learning and educational innovation at the University of Santiago de Compostela: strategies for institutionalization
}

\begin{abstract}
Service learning constitutes a proposal of educational innovation that optimizes the teaching-learning processes, favors the acquisition of professional competences and the strengthening of a civic-social commitment with the community. The University of Santiago de Compostela (USC) has initiated the process of institutionalization of the service learning, contributing to the consolidation and diffusion to different experiences that have gradually been emerging. The present work describes this development, emphasizing teacher training, educational innovation projects and the dissemination and recognition as the three pillars that support the strategy of institutionalization of service learning in the USC. The results obtained so far show the interest of a significant number of teachers on this methodology and its contribution to students education.
\end{abstract}

\section{Keywords}

Service learning, educational innovation, teaching, university, institutionalization. 


\section{Introducción}

Desde los inicios del proceso de convergencia y adaptación al Espacio Europeo de Educación Superior (EEES), conceptos como innovación educativa y aprendizaje servicio (ApS) han comenzado a estar presentes en los discursos y planes estratégicos de las instituciones de educación superior. $\mathrm{Si}$ bien cada uno de ellos referenciado y definido de diferente manera en función de la institución y el contexto, ambos conceptos hoy en día caminan de la mano y están cada vez más presentes en los documentos que recogen la misión y valores de nuestras universidades (UNESCO, 2009; Ministerio de Educación, 2010, 2011; CADEP, 2012; CRUE, 2015).

Si por innovar se entiende "no solo mejorar un proceso o un producto sino que implica generar un verdadero cambio" (Gros y Lara, 2009, 225), la innovación se interpreta como un proceso que conlleva la introducción de nuevos elementos de valor contrastado que favorecen el desarrollo de un proyecto de mayor calidad. Así, la innovación vinculada a la educación supone asumir riesgos en el diseño de nuevos caminos para obtener resultados que conllevan una diferenciación y mejora de la calidad del proyecto de nuestra universidad en relación con las del entorno.

La universidad constituye un contexto propicio para el desarrollo de competencias cívicas y sociales (Santos y Lorenzo, 2007; Villa y Villa, 2007; Martínez, 2008; Naval 2008; Ugarte y Naval, 2010; Lorenzo, 2012; Sotelino,
2015). En este sentido, el EEES reformula el concepto de educación superior añadiendo como fundamentales contenidos relacionados con la ética, los valores y las competencias transversales, difíciles de explicitar en un espacio curricular tradicional. Se trata de implementar, pues, una innovación metodológica que favorezca la incorporación de contenidos de compromiso cívico en el desarrollo curricular.

Es aquí, en esta confluencia de principios, innovación educativa y competencias cívico-sociales donde la institucionalización de la metodología del ApS cobra sentido, entendiendo tal como recoge Puig $(2009,9)$ :

\footnotetext{
"Metodología que combina en una sola actividad el aprendizaje de contenidos, competencias y valores con la realización de tareas de servicio a la comunidad. En el aprendizaje servicio, el conocimiento se utiliza para mejorar algo de la comunidad y el servicio se convierte en una experiencia de aprendizaje que proporciona conocimiento y valores".
}

Ya inmersos en el proceso de desarrollo del EEES, son varios los autores (Boyle, 2007; Newman, 2008; Francisco y Moliner, 2010; Rodríguez Gallego, 2013; Glazier, Able y Charpentier, 2014; Aramburuzabala, Cerillo y Tello, 2015; Santos, Sotelino y Lorenzo, 2015; Saz y Ramo, 2015; Sotelino, 2015; Gil et al., 2016) que avalan la importancia de la participación del alumnado en proyectos de aprendizaje servicio, de cara a la formación de estudiantes más implicados social, política y profesionalmente, con la idea de una sociedad más justa y comprometida, críticos con la misma y capaces de contribuir a su desarrollo sostenible. 
No obstante, la institucionalización del ApS en las universidades comporta la presencia de una masa crítica que favorezca y lidere su implantación, pero también de un apoyo institucional que le proporcione el necesario soporte, tal y como recogen Santos, Sotelino y Lorenzo $(2015,76)$ :

"No hemos de pretender que el aprendizaje servicio resuelva todos los problemas, ni mucho menos. Lo que si implica es una muestra de pedagogía experiencial, que con poca inversión económica, puede optimizarlos procesos de enseñanza-aprendizaje en consonancia con la declaración de Bolonia. Y aunque la meta pueda ser razonable, no basta con que algunos profesores asuman la encomienda de abanderar experiencias. Se necesita un compromiso institucional con la responsabilidad social y con una mejor formación del alumnado, y más si se quiere avanzar con pasos seguros en época teñida de tanta incertidumbre".

\section{Modelo de institucionalización del ApS en la USC}

El auge del ApS en la Educación Superior está ligado a una activa relación de la universidad con la sociedad civil. La CRUE (2015) reconoce la importancia de la institucionalización del ApS como estrategia docente dentro del marco de la Responsabilidad Social Universitaria para la promoción de la sostenibilidad, en particular como herramienta clave para impulsar la sostenibilización curricular, a fin de contribuir al desarrollo de una sociedad más justa y mejorar los aprendizajes académicos y sociales que favorezcan el desarrollo competencial y el futuro desempeño profesional del alumnado.

La USC, consciente de esta demanda, está inmersa en un proceso de desarrollo de la plena institucionalización del ApS, que se asienta en el marco de lo señalado en su Plan Estratégico 2011-2020 (USC, 2011) sobre el compromiso y la implicación con la sociedad, como una línea de acción que incluye entre sus objetivos "reforzar el compromiso de la USC con la responsabilidad social y aumentar la sensibilización y la participación de los miembros de la comunidad universitaria en proyectos relacionados con el voluntariado, la participación social y la cooperación al desarrollo" (86-87). Por otra parte, establece como un elemento de mejora el fomento de una metodología docente que promueva una "mayor participación del estudiantado y una necesidad de transmitirle no solo conocimientos sino también habilidades y competencias" (40).

Con el objetivo general de impulsar el ApS en la USC, se ha implementado un plan de actuación, apoyado en las experiencias previas existentes en ApS $y$ en un creciente acervo de investigación, que persigue situarse en el logro de una institucionalización sostenible en la línea señalada por Furco (2011). A tal fin, se estructuran tres ejes funcionales y complementarios: la formación del profesorado, los proyectos de innovación educativa en ApS y el reconocimiento y la difusión (figura 1 ). 


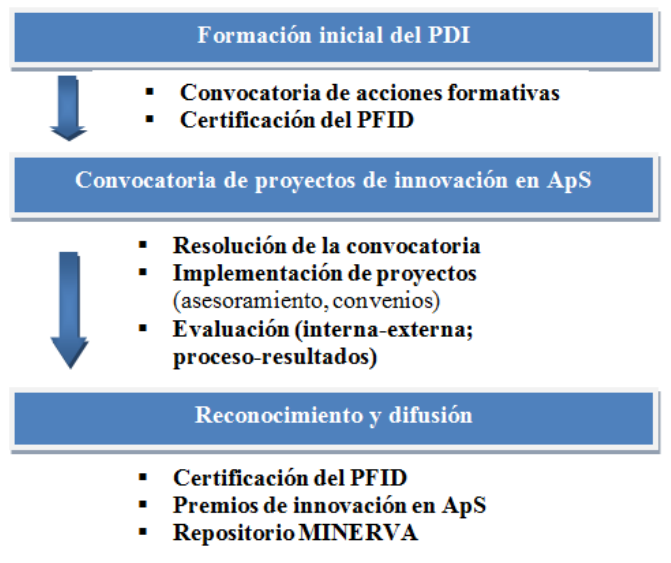

Figura 1. Plan de acción del ApS en la USC.

\subsection{Formación inicial}

La formación constituye el punto de partida necesario para dar a conocer el ApS como una metodología docente innovadora que permita generar comunidades comprometidas y promotoras en la implementación y apoyo al ApS. En esta línea, desde el Programa de Formación e Innovación Docente (PFID) se vienen desarrollando, desde el año 2012, acciones formativas en donde se abordan cuestiones relativas a la consecución de cuatro bloques de objetivos básicos:

-Sensibilizar, motivar y predisponer al profesorado hacia el ApS.

-Proporcionar una base conceptual y metodológica.

-Ayudar, descubrir y explorar posibles ámbitos de aplicación del ApS en las materias, provocando una reflexión sobre y desde la propia práctica docente.

-Dar pautas para el diseño, implementación y evaluación de un proyecto concreto de ApS.

\subsection{Proyectos de innovación educativa en ApS}

Los proyectos de innovación en ApS tienen por objeto impulsar, asesorar, reconocer y dar difusión a iniciativas de innovación educativa que utilicen la metodología de ApS. Su finalidad es mejorar los procesos de enseñanzaaprendizaje mediante la integración de la preparación para la práctica profesional y para el ejercicio de la responsabilidad social del alumnado de la USC, en el marco de las diferentes materias de grado y posgrado. Su desarrollo se vertebra en torno a tres grandes momentos:

a)Convocatoria. De carácter anual, se dirige al conjunto de personal docente e investigador, con vinculación en su plan de ordenación académica para la materia o materias objeto del proyecto. Las iniciativas pueden ser de carácter individual o grupal y vinculadas a una o varias asignaturas, con la intencionalidad de englobar también aquellas proposiciones de carácter interdisciplinar.

Para la selección de las propuestas, se valoran los siguientes criterios: la idoneidad del proyecto para la consecución de los objetivos de la convocatoria (pertinencia), su alcance respecto a la experiencia innovadora propuesta (relevancia), la posibilidad de implementación (viabilidad), la justificación en base a las necesidades detectadas, la articulación, claridad y concrección de los diferentes 
componentes del proceso (coherencia y consistencia), la posibilidad de su generalización y de llegar a convertirse en una práctica docente estable (proyección) y la originalidad de la propuesta.

b)Implementación. Una vez resuelta la convocatoria de proyectos, comienza la fase de desarrollo. En esta etapa, se establecen tres momentos diferenciados:

-Asesoramiento y evaluación inicial. Este paso corresponde a la evaluación del diseño el cual se realiza a través de la valoración y asesoramiento de expertos en ApS (criterio interjueces). Su finalidad es emitir un juicio sobre la suficiencia y adecuación de la propuesta con el objetivo de redefinir y mejorar el proyecto.

-Formulación de convenios.

Corresponde al SEPIU la gestión de los protocolos de colaboración con las entidades implicadas en la realización del servicio.

-Seguimiento y tutorización. Durante todo el período de puesta en marcha, cada proyecto cuenta con el acompañamiento de un tutor o tutora que realiza las funciones de asesoría, seguimiento y evaluación. La formación que adquiere el profesorado durante esta fase es reconocida con 10 horas por el PFID.

c)Evaluación. Está centrada tanto en el proceso como en los resultados y contempla procedimientos de evaluación interna y externa. Algunas de las estrategias para la evaluación de la implementación son la elaboración de informes de autoevaluación por parte de los implicados en el programa (alumnado, profesorado, partners) y la entrevista de grupo con el alumnado participante. Por otro lado, para la evaluación de los resultados se hace uso de escalas de aplicación al alumnado sobre competencias cívicosociales y de cuestionarios de satisfacción al alumnado y al profesorado. Al finalizar el proyecto, el profesor coordinador debe remitir una memoria para su valoración por parte de la comisión.

\subsection{Reconocimiento y difusión}

Con la finalidad de motivar, visibilizar y otorgar reconocimiento a la innovación educativa en ApS se establecen diferentes acciones, que podrían sintetizarse en:

- Certificaciones de participación en la formación y en el desarrollo de proyectos de innovación.

-Convocatoria anual de Premios de innovación educativa en ApS, con el objeto de reconocer aquellos proyectos que, por sus características y trayectoria, son de carácter sobresaliente.

-Difusión de los proyectos desarrollados, a modo de buenas prácticas, a través del repositorio insitucional MINERVA.

La institucionalización del ApS no sólo ha demandado la existencia de un plan de actuación, sino también que ha introducido sinergias importantes entre 
diferentes servicios y ha conformado alianzas estratégicas que contribuyen a la integración de las diferentes iniciativas de ApS en la estructura de la Universidad. Así, destacar la dinámica colaborativa entre el Servicio de Participación e Integración Universitaria (SEPIU), el Programa de Formación e Innovación Docente (PFID) y el Grupo de Investigación ESCULCA.

\section{EI Papel del Servicio de Participación e Integración Universitaria (SEPIU) en la promoción del ApS en la USC}

\section{El trabajo que la Universidad de} Santiago de Compostela viene desarrollando en la promoción de la participación social y del voluntariado, entre los miembros de la comunidad universitaria, ha posibilitado un conocimiento exhaustivo de las necesidades de las entidades de acción social del entorno próximo de sus dos campus. A día de hoy estas colaboraciones se extienden incluso a los municipios adyacentes o a ciudades más alejadas de los campus de la USC.

El conocimiento que un servicio como el SEPIU ha acumulado sobre las necesidades y actividades de las entidades, así como el trabajo desarrollado por los gestores y voluntariado supone una oportunidad para el desarrollo de proyectos de ApS, ya que pone a disposición del profesorado la intermediación necesaria con entidades colaboradoras.

La USC cuenta actualmente con convenios marco de colaboración con más de 70 entidades, de un amplio abanico de campos de actuación, que desarrollan 175 programas distintos por toda la Comunidad Autónoma de Galicia.

A la hora de iniciar un proyecto de ApS es fundamental la búsqueda de socios, por lo que contar con la colaboración de un servicio propio de la universidad que valore y asesore sobre las posibilidades de trabajo con una entidad u otra, será fundamental para informar y orientar al profesorado en el diseño e implementación de un proyecto de innovación en ApS.

En la mayoría de los casos, ya existe un convenio marco de colaboración con las entidades. De no ser así, el propio servicio se encarga de realizar los trámites necesarios (análisis de la entidad, validación de los proyectos, verificación de la validez de sus propuestas, etc.) para poder llegar a formalizarlo.

Otra cuestión relevante es el seguro para el alumnado participante en este tipo de proyectos, que debe ser diferente del seguro escolar. La formalización del seguro ha de realizarse mediante una póliza específica, que en el caso de la USC está abierta para el voluntariado y el alumnado que lleva a cabo actividades de participación social.

Así pues, el SEPIU, al igual que con los participantes de cualquier proyecto de voluntariado, se encarga de la gestión del seguro de cada uno de los estudiantes que participan en la experiencia de ApS. De este modo, se da respuesta a uno de los problemas 
que más tienden a preocupar al profesorado a la hora de implementar un proyecto de ApS, la cobertura con un seguro de su alumnado fuera del horario académico mientras colaboran en la experiencia de aprendizajeservicio.

\section{Formación e innovación educativa en ApS: algunos resultados}

El seguimiento del plan de actuación permite ofrecer datos que ilustran el desarrollo del proceso de institucionalización del ApS en la USC en el momento actual, tanto en el campo de la formación del profesorado como de los proyectos de innovación educativa.

\subsection{Formación}

En el marco definido por el Programa de Formación e Innovación Docente (PFID), cuyo objetivo radica en establecer un espacio de formación que facilite la adquisición y mejora de competencias docentes, investigadoras y de gestión, se han celebrado desde el año 2012 diferentes actividades formativas sobre el ApS. En total se contabilizan, hasta el curso 2015-2016, un total de 7 cursos ( 3 de ellos en el Campus de Lugo y 4 en el de Santiago).

En el conjunto de las acciones

formativas han participado 103 docentes de ambos campus (71 en Santiago y 32 en Lugo) (tabla 1), de los cuales el $59 \%$ son profesoras y el $41 \%$ profesores (gráfico 1 ).

En el curso académico 2014-2015, se ha celebrado el I Simposio Internacional Aprendizaje-Servicio en la Educación Superior: Innovación, Calidad e Institucionalización, que fue reconocido como actividad formativa para el profesorado de la USC.

\begin{tabular}{|c|c|c|c|c|c|c|c|}
\cline { 3 - 8 } \multicolumn{2}{|c|}{} & 2011-2012 & 2012-2013 & 2013-2014 & 2014-2015 & 2015-2016 & Total \\
\hline $\begin{array}{c}\text { No } \\
\text { matriculados/ass }\end{array}$ & Santiago & 30 & 9 & 12 & - & 20 & 71 \\
\cline { 2 - 8 } & Lugo & - & 9 & 13 & - & 10 & 32 \\
\hline \multicolumn{2}{|c}{ Total } & 30 & 18 & 25 & - & 30 & 103 \\
\hline
\end{tabular}

Tabla 1. Número de matriculados/as en cursos de formación en ApS

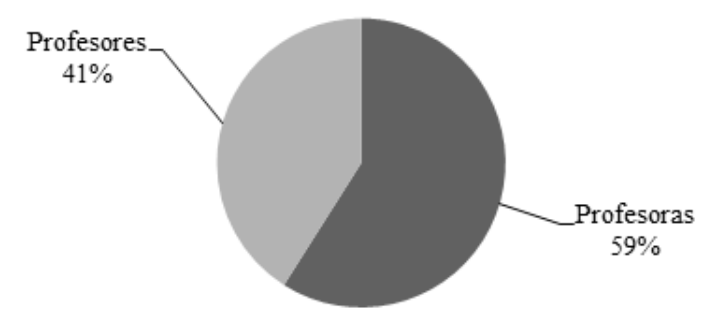

Gráfico 1. Distribución de los participantes por género

La distribución por áreas de conocimiento (gráfico 2) muestra una mayoría de participantes de Ciencias Sociales y Jurídicas (30\%), seguido de Ciencias Experimentales (24\%), Humanidades (19\%), Ciencias de la Salud $(16 \%)$ y Enseñanzas Técnicas $(11 \%)$. 


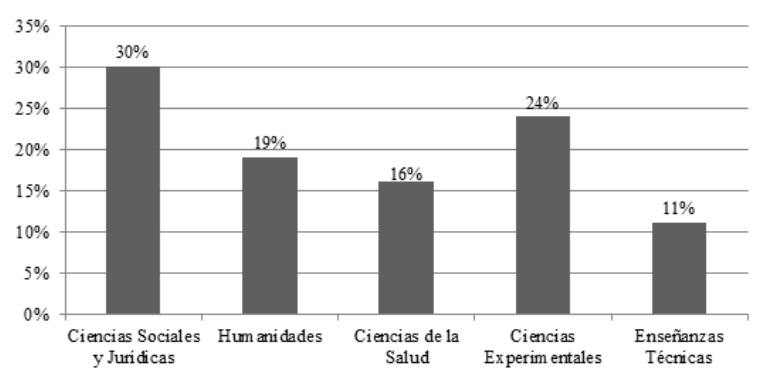

Gráfico 2. Distribución de los/as participantes por áreas de conocimiento

\subsection{Proyectos de innovación educativa en ApS}

En el curso 2015-2016, la USC establece la I Convocatoria de Proyectos de Innovación Educativa en ApS, a la que se presentaron un total de 21 solicitudes, 15 enmarcadas en el Campus de Santiago y 6 de ellas impulsadas por profesorado del Campus de Lugo. La gran mayoría son de carácter grupal $(\mathrm{N}=15)$ y toman como marco materias de Grado $(\mathrm{N}=19)$, mientras que sólo uno de los proyectos se desarrolla en el nivel de Máster. Además, otra de las iniciativas, de carácter interdisciplinar, vincula la propuesta a una asignatura de Grado y otra de Máster (tabla 2).

\begin{tabular}{|l|l|c|c|}
\hline & & N & $\%$ \\
\hline \multirow{3}{*}{ Campus } & Lugo & 6 & $28,6 \%$ \\
\cline { 2 - 4 } & Santiago & 15 & $71,4 \%$ \\
\hline \multirow{3}{*}{ Titulación } & Grado & 19 & $90,5 \%$ \\
\cline { 2 - 4 } & Máster & 1 & $4,7 \%$ \\
\cline { 2 - 4 } & Gradoy Máster & 1 & $4,7 \%$ \\
\hline \multirow{3}{*}{ Carácter } & Individual & 6 & $28,6 \%$ \\
\cline { 2 - 4 } & Grupal & 15 & $71,4 \%$ \\
\hline
\end{tabular}

Tabla 2. Solicitudes presentadas a la I Convocatoria de proyectos de innovación educativa en ApS
El grado de participación en la convocatoria por áreas de conocimiento ha sido desigual, siendo el profesorado de Ciencias Sociales y Jurídicas el que ha presentado un mayor número de proyectos $(\mathrm{N}=13)$. Con una presencia inferior se encuentran Ciencias de la Salud $(\mathrm{N}=3)$, Enseñanzas Técnicas $(\mathrm{N}=2)$, Humanidades $(\mathrm{N}=1)$ y Ciencias Experimentales $(\mathrm{N}=1)$. Además, del conjunto de solicitudes recibidas, una de ellas realizaba una propuesta que aglutinaba materias de dos áreas:

Ciencias Sociales y Jurídicas y Enseñanzas Técnicas (gráfico 3).

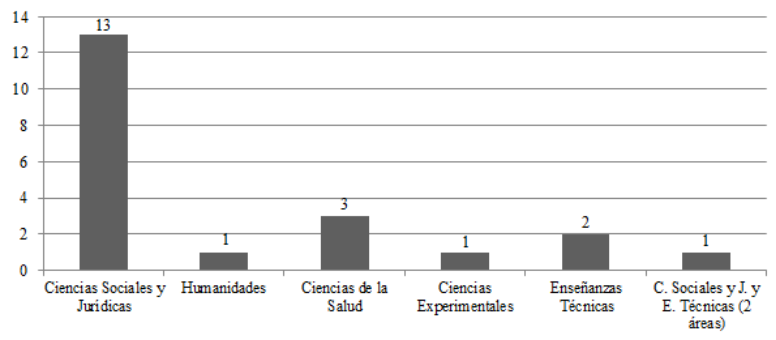

Gráfico 3. Distribución de las solicitudes por áreas de conocimiento

\section{Conclusiones y desafíos}

En línea con el trabajo ya iniciado en otras universidades (Marquès, 2014; Jouannet et al., 2015), el proceso de institucionalización del ApS en la USC es el resultado de una senda dirigida a asentar y consolidar diversas iniciativas individuales y colectivas, dando sistematicidad y legitimación a prácticas, experiencias y acciones de formación y de divulgación que ya se venían desarrollando en su seno.

Durante los últimos años, un número relevante de profesorado ha participado en las actividades formativas 
organizadas sobre la temática, en el marco del PFID de la USC, lo que denota su interés por conocer las posibilidades de la metodología del ApS para la docencia. Al tiempo, estas acciones han propiciado el impulso de grupos de trabajo, así como la emergencia de liderazgos positivos para su difusión.

La convocatoria de proyectos ha propiciado dar visibilidad y reconocimiento a experiencias con una amplia trayectoria. Del mismo modo, ha supuesto el surgimiento de nuevas iniciativas de gran interés en ámbitos disciplinares emergentes. El proceso de institucionalización ha comportado el acompañamiento, asesoría y evaluación de los proyectos favoreciendo procesos de formación y mejora.

La existencia de estructuras consolidadas dentro de la USC destinadas a la formación e innovación docente (PFID) y a la promoción de la participación social y el voluntariado (SEPIU) han sido las bases que han hecho posible articular el marco institucional y organizativo para la implementación y desarrollo del ApS, a lo que hay que añadir el contar con un amplio acervo en el terreno de la investigación de grupos especializados en este campo, como el Grupo ESCULCA, de gran relevancia para el asesoramiento y la formación.

El disponer, desde hace más de veinte años, del SEPIU ha permitido a nuestra Universidad desarrollar una importante red de alianzas con agentes e instituciones del tercer sector, que entendemos como fundamentales para el desarrollo de los proyectos de ApS en las universidades, a la vez que facilita al profesorado la búsqueda de socios entre las diferentes instituciones del entorno. El conocimiento de la implicación de la universidad en la promoción de la participación social y del voluntariado por parte de los distintos agentes (sociales, culturales, económicos, etc.) favorece el desarrollo de las sinergias necesarias para dar el paso desde los proyectos con voluntarios a los proyectos de ApS dentro de las entidades.

Esta realidad nos sitúa frente al desafío de continuar dando profundidad al proceso de institucionalización del ApS en la USC, en particular en lograr una mayor alineación con la misión institucional de la Universidad y una mayor presencia en los documentos de planificación estratégica.

\section{Bibliografía}

Aramburuzabala, P., Cerrillo, R. y Tello, I. (2015). Aprendizaje-servicio: una propuesta metodológica para la introducción de la sostenibilidad curricular en la universidad.

Profesorado, Revista de Currículum y Formación del Profesorado, 19(1), 7895.

Boyle, M. E. (2007). Learning to neighbor? Service-learning in context. Journal of Academic Ethics, 5, 85-104.

CADEP (2012). Directrices para la introducción de la sostenibilidad en el currículum. Recuperado de http://angelsull.es/sostenibilidad/wpcontent/uploads/2013/04/Directrices- 
Sostenibilidad-curriculum-CRUE.pdf

CRUE (2015). Institucionalización del Aprendizaje-Servicio como estrategia docente dentro del marco de la Responsabilidad Social Universitaria para la promoción de la Sostenibilidad en la Universidad. Recuperado de http://www.crue.org/Documentos $\% 20$ c ompartidos/Recomendaciones $\% 20 y \% 2$ 0criterios\%20tecnicos/2.\%20APROBAD A\%20INSTITUCIONALIZACION\%20ApS .pdf

Francisco, A. y Moliner, L. (2010). El Aprendizaje Servicio en la Universidad: una estrategia en la formación de la ciudadanía crítica. REIFOP, 13(4), 6977.

Furco, A. (2011). Rúbrica de autoevaluación para la institucionalización del aprendizajeservicio en la educación superior (revisión 2003). Educación Global Research, 0, 77-88. Recuperado de http://educaciocp89.webjoomla.es/wpcontent/uploads/04-Furco-2Castellano.pdf

Gil, J., Moliner, O., Chiva, O. y García, R. (2016). Una experiencia de aprendizaje-servicio en futuros docentes: desarrollo de la competencia social y ciudadana. Revista Complutense de Educación, 27(1), 5373.

Glazier, J.; Able, H. y Charpentier, A. (2014). The impact of Service-Learning on Preservice Profesionals' Dispositions Towards Diversity. Journal of Higher Education Outreach and Engagement,
18(4), 177-198.

Gros, B. y Lara, P. (2009). Estrategias de innovación en la educación superior: el caso de la Universitat Oberta de Catalunya. Revista Iberoamericana de Educación, 49, 223-245

Jouannet, C.; Montalva. J.T.; Ponce, C. y Von Borries, V. (2015). Diseño de un modelo de institucionalización de la metodología de aprendizaje servicio en educación superior. RIDAS, Revista Iberoamericana de Aprendizaje Servicio, 1, 112-131.

Lorenzo, M. (2012). La función social de la universidad y la formación del profesorado. Edetania: estudios y propuestas socio-educativas, 42, 25-38

Marquès, M. (2014). La dimensión docente de la Responsabilidad Social Universitaria: la institucionalización del aprendizaje servicio en la Universitat Rovira i Virgili. I Jornadas Internacionales sobre Responsabilidad Social Universitaria. Cádiz, 20-21 de febrero de 2014. Recuperado de http://www.urv.cat/media/upload/arxiu s/aprenentatgeservei/Jornades $\% 20 i \% 20$ congressos $\% 2$ OAPS/MMarques-\%20RSU\%20$\% 20 A P S . p d f$

Martínez, M. (ed.) (2008). Aprendizajeservicio y responsabilidad social de las universidades. Barcelona: Octaedro.

Ministerio de Educación (2010). Estrategia Universidad 2015. Contribución de las universidades al progreso socioeconómico español 20102015. Recuperado de 
http://www.educacion.gob.es/eu2015/I a-eu2015.htm

Ministerio de Educación (2011). La responsabilidad social de la universidad $y$ el desarrollo sostenible. Recuperado de

https://sede.educacion.gob.es/publiven $\mathrm{ta} / \mathrm{d} / 14925 / 19 / 0$

Naval, C. (2008). Universidad y conciencia cívica. Algunas experiencias fructíferas: service-learning y campus compact. Revista Sembrando Ideas, 2, 57-80.

Newman, J. (2008). Service learning as an expression of ethics. New Directions for Higher Education, 142, 17-24.

Puig, J.M. (2009). Aprendizaje servicio (ApS): educación y compromiso cívico. Barcelona: Graó.

Rodríguez Gallego, M. (2013). El Aprendizaje-Servicio como estrategia metodológica en la Universidad. Revista Complutense de Educación, 25(1), 95113.

Santos, M. A., Sotelino, A. y Lorenzo, M. del M. (2015). Aprendizaje-servicio y misión cívica de la universidad. Una propuesta de desarrollo. Barcelona: Octaedro.

Santos, M.A. y Lorenzo, M del M. (2007). Universidade e construción da sociedade civil. Vigo: Edicións Xerais.

Saz, Isabel y Ramo, R.M. (2015). Aproximación a los impactos y beneficios del aprendizaje servicio en la Universidad de Zaragoza. RIDAS, Revista Iberoamericana de Aprendizaje
Servicio, 1, 9-27.

Sotelino, A. (2015). Aprendizaje servicio y educación superior en Galicia. ¿Qué opinan los profesores? RIDAS, Revista Iberoamericana de Aprendizaje Servicio, 1, 56-75.

Ugarte, C. y Naval, C. (2010).

Desarrollo de competencias profesionales en la educación superior. Un caso docente concreto. Revista electrónica de Investigación Educativa, 12, 1-14. Recuperado de redie.uabc. $\mathrm{mx} /$ index.php/redie/article/v iew/251/412

UNESCO (2009). Conferencia Mundial sobre la Educación Superior 2009: La nueva dinámica de la educación superior y la investigación para el cambio social y el desarrollo.

Recuperado de http://www.unesco.org/education/WCH E2009/comunicado_es.pdf

USC (2011). Plan Estratéxico 20112020. Universidade de Santiago de Compostela. Recuperado de http://www.usc.es/export9/sites/webin stitucional/gl/goberno/vrcalidade/desca rgas/proplanestrUSC_2011-20.pdf

Villa, A. y Villa, O. (2007). El aprendizaje basado en competencias y el desarrollo de la dimensión social en las universidades. Educar, 40, 15-48. 